\title{
Da Epistemologia dos Estudos de Defesa e os seus Campos Híbridos
}

\section{From the Epistemology of Defense Studies and its Hybrid Fields}

SABRINA EVANGELISTA MEDEIROS

\section{INTRODUÇÃO}

Com a ascensão de estudos que têm a defesa como tema principal entre acadêmicos brasileiros de variadas gerações nos últimos 10 anos, cabe pensar qual o lugar que ocupa este campo de conhecimento em origem, estrutura e métodos de classificação. Cabe observar a construção deste campo e, em especial, no Brasil, para que a consciência sobre o lugar em que se encontra seja compatível com o que representa como interesse nacional e diante dos demais campos de conhecimento correlatos.

Este artigo volta-se ao tema dos estudos de defesa, que nem sempre são assim nomeados, suas demarcações enquanto campo do conhecimento, suas variações e indagações a respeito de uma área de crescente importância. Minha postulação decorre da hipótese da presença de campos híbridos, não objetivamente qualificados e, certamente, maiores do que as definições normativas no Brasil podem abrigar. Embora a epistemologia enquanto conceito trate da relação entre o ser indagador e o objeto inerte, a nossa premissa parte da concepção de que é a epistemologia regida pela natureza, etapas e limites do conhecimento, o que faz dela dinâmica e variável em seu resultado.

Portanto, apresentaremos as razões e incertezas do campo da defesa em sua configuração teórica e a partir dos seus temas e objetos constituintes. De outro modo, localizaremos como se configurou entre políticas e modelos institucionais. Por fim, iremos propor uma análise que configure um espaço onde hierarquias e o núcleo operativo do campo não destituam

Sabrina Evangelista Medeiros - Doutora em Ciência Política (IUPERJ) e professora de Estudos Estratégicos (EGN). E-mail: sabrinamedeiros@yahoo.com. 
a existência dos aqui nomeados campos híbridos, detectados a partir de regiões interdisciplinares igualmente relevantes para a caracterização do crescimento do interesse e número de pesquisadores na área.

\section{DA EPISTEMOLOGIA E DA FORMAÇÃO DE UMA COMUNIDADE EPISTÊMICA EM DEFESA}

Utilizando-me de Schopenhauer (1819; trad. 2001), procurando atribuir algum ceticismo às minhas origens rawlsianas (Rawls, 1971) e institucionalistas, parto do pressuposto que a vontade humana é regente, contudo, incapaz de conscientemente abrigar aquilo que é formado pelas condições externas e intraduzíveis ao homem em conjunto com suas escolhas. Isso porque há algo de inevitável no que vemos nos últimos anos no campo da defesa, entre o que fizemos e do que vimos construir-se. Há algo entre o que conscientemente fizeram os atores e o que se modificou pela conjuntura externa, pelas necessidades institucionais, pela evolução natural das instituições.

Pois se abstrairmos inteiramente daquele mundo como representação propriamente dito, nada resta além do mundo como vontade. A vontade é o em-si da ideia, esta objetivando perfeitamente aquela; ela também é o em-si da coisa individual e do indivíduo que conhece esta; estes objetivando imperfeitamente aquela (Schopenhauer, 2001, p. 23).

O pressuposto que aqui reside é o de que ações são combinadas às reações, previstas e não previstas, causando evolução constante dos interesses e instituições. Desse modo, partimos com base em uma visão funcionalista (Parsons, 1975) do campo dos estudos de defesa, no que diz respeito à configuração parcialmente vinculada ao que deveria ser e parcialmente ao que se tornou de fato, cuja crença está em um movimento contínuo e gradual. Também reverbera aqui a crença no projeto institucional em curso, estruturante, e na vinculação deste projeto aos padrões históricos inexoráveis a cada processo, de modo que importam as instituições criadas ao longo e os seus mecanismos regulatórios.

Para isso, alguns elementos são determinantes para esta abordagem. Primeiramente, o processo de criação deste campo é entendido por meio de uma análise macrossociológica. Segundo, as diferentes partes do processo contribuem para a formação do campo, ou seja, há uma função para cada parte. Terceiro, a interdependência entre estas partes gera o equilíbrio que se quer, por exemplo, na existência de um campo de estudos. Por último, a consideração de que é este campo formado por elementos de ordem evolucionária, onde a complexidade é predominante e subsistemas 
e sistemas vão adaptando e sendo adaptados progressivamente (Turner, 1991; Wallace e Wolf, 1995).

Tal combinação entre uma evolução funcional do campo da defesa e sua evolução institucional gradual, na qualidade de regras, normas, área ou editais, nos parece ser a configuração mais próxima entre a demanda, os resultados e os movimentos de ordem social que permeiam o processo, como da aproximação entre o mundo civil e o mundo militar.

Portanto, esta não foi constituída como uma fala completa ou objetiva, posto que dotada de uma observação tão particular quanto a minha - também regida pelo princípio da vontade de Schopenhauer, que limita a minha representação do mundo ordinário. Além disso, está em jogo a minha particular experiência como fruto da ascensão deste campo, tal como escolhi observá-lo. O que se enuncia é uma observação livre e filosófica, passível de ser falseada e refutada, em seus princípios, dados de observação e consequências (Popper, 1972; Diniz, 2010).

Assim, quando da manifestação de uma nova área de conhecimento, observando-se a natureza mutante do próprio conhecimento por meio de uma abordagem evolucionária da epistemologia (Popper, 1972), é natural que a organização dos espaços demande também a reorganização dos recursos, baseada nas novas formas de disposição das necessidades humanas. Dentro disso, há algo de intrínseco, particular do universo cultural ou político onde se está inserido - brasileiro, regional, federativo, municipal - assim como há algo de universal, algo a ser observado em outras realidades e experiências.

Neste último caso, as experiências internacionais devem ser observadas com vistas à participação e relevância desta comunidade epistêmica, que sempre irá dispor de algo inusitado e relevante. Por outro lado, dadas as particularidades de cada comunidade epistêmica, há de se reconhecer que mecanismos nacionais próprios devem ser construídos para que os interesses espontaneamente criados sejam respaldados por e para esta comunidade.

A este respeito, Peter Haas publicou um artigo seminal sobre comunidades epistêmicas e política internacional (Haas, 1992). É interessante notar que o artigo trazia o tema das incertezas e complexidade progressiva para contribuir com a construção de um campo que era composto por meio das relações internacionais e que fazia prevalecer o interesse crescente nas instituições, embora o campo se dividisse entre crédulos e incrédulos nelas. Os problemas das relações internacionais pareciam mais voltados do que antes à capacidade de manutenção do consenso sobreposto induzido por Rawls (1971) anos antes de Haas, entre atores estatais, crescentemente movidos por atores de outra ordem. 
Neste mesmo texto, Haas ressalta que o uso que faz do conceito de comunidade epistêmica está em algum lugar entre o limitado conceito das ciências naturais e aquele usado por alguns dos mais tradicionais filósofos, como Karl Marx que, segundo Peter Haas, voltava-se para a defesa de uma comunidade que tem as mesmas crenças em métodos e na construção de uma verdade.

Do contrário, em sua definição, as comunidades epistêmicas seriam organizadas em algum grau de racionalidade, impresso na ordem entre causas e consequências, onde a observação garantiria uma ação social comum em busca de resultados e do desenho de soluções para os problemas detectados. Se transnacionais, estas comunidades epistêmicas teriam capacidade de provocar novos e renovados tipos de relações interestatais.

Muito embora o texto de Haas também proponha uma subdivisão dentre os modelos de compreensão das Relações Internacionais, que incluiriam ainda os neorrealistas e os pós-estruturalistas, as comunidades epistêmicas, tal como apresenta, reuniram características que transpassariam profissões (no sentido de formações, tal como a de economista) e que reuniriam grupos e subdivisões voltados para os mesmos problemas.

\section{DA NATUREZA E FONTES DE CONHECIMENTO DO CAMPO}

A começar pela natureza do campo, é possível notar que estudos relacionados à defesa são comumente identificados por nomenclaturas mais antigas do que a pesquisa em defesa (defense research), tal como as ciências militares (military sciences), os estudos militares (military studies), os estudos estratégicos (strategic studies). Em todas estas definições há elementos de interdisciplinaridade que podem ser observados à luz das ciências puras e aplicadas ou das ciências humanas e sociais. É possível dizer-se, inclusive, que não há um só estudo de defesa que não prescinda de teoria política, das relações internacionais ou da sociologia, em combinação com métodos e objetos provenientes da economia, do estudo dos conflitos, da administração.

No entanto, embora determinantes porque pertencentes às relações estatais, os estudos de defesa pouco foram constituídos por projetos que combinam as ciências exatas a todas áreas supracitadas. Isso não quer dizer que projetos de defesa de natureza das ciências exatas não contribuam com a composição do campo; apenas sua interdisciplinaridade é restrita, porque distintas em nível: o técnico e o estratégico. Decerto, quanto mais técnico, mais determinado por fronteiras observáveis, ainda que também dotadas de interdisciplinaridade. Além disso, no que diz respeito às questões de ordem técnica, a presença de estudos de caráter sigiloso é mais presente nes- 
ta área do que em outras das ciências duras, da saúde ou da natureza. Isso porque enquanto a inovação rege a tecnologia, na defesa ela está sujeita ao interesse nacional maior do que a obtenção de patentes pode representar (Moreira, 2011).

Neste âmbito, os estudos estratégicos formaram a grande produção do campo, comumente voltada aos estudos da guerra e da paz. Neste caso, não só o assunto era privilégio e prioridade de instituições militares, como também o seu escopo voltado para os períodos de enfrentamento - frutos do estabelecimento, ampliação geopolítica e de sobrevivência dos Estados - assim como os períodos de recuperação e preparação para a guerra. O lócus parte do tema da guerra e a fronteira é assim determinada pelas necessidades subjacentes a ela.

O problema reside no reconhecimento de que, não raro, estudos interdisciplinares sofrem do efeito de subcampos disciplinares. Neste sentido, as disciplinas tradicionais como a ciência política ou as engenharias ainda representam os estudos de defesa de forma limitada justamente porque tal dicotomia e separação não representa as conexões entre estes campos. Talvez, de fato o subcampo exista como área de especialidade, mas a hierarquia que reforça na composição de um subcampo prejudica ainda mais a visualização de sua representação como fonte da conexão entre áreas principais e objetivamente delimitadas.

Isso porque a interposição de campos e disciplinas não é comumente representada em redes complexas - aqui proponho uma representação multidimensional baseada em mapa, cuja representação de campos híbridos se dá por meio de clusters de proximidade. Trazendo os estudos epistemológicos para as necessidades interpostas neste debate, alinharíamos a pergunta sobre qual a melhor maneira de observar como a realidade pode ser entendida e como ela tem sido representada.

Considerando-se esta disposição em rede, o primeiro dos vínculos ocorreria entre os estudos estratégicos e as áreas que estruturam as componentes necessárias à guerra. Tal conceito é observado neste momento à luz do vínculo tradicional com as estruturas militares disponíveis ao enfrentamento interestatal clássico, ao conflito intraestatal ou de ordem civil, à guerra irregular, ou à guerra por recursos - esta última, entendida como a disposição de manter reduzida a vulnerabilidade estatal quanto à ausência de energia, minerais variados, alimento, água.

Procurando organizar em estruturas de conhecimento esta rede, ao começar pela guerra, lateralmente podemos nos remeter aos subsídios à guerra ou aos resultados dela. São componentes desta face das relações o estudo dos materiais bélicos, das componentes militares; da tecnologia militar. Como uma das intersecções com a administração, a logística, ainda 
com as engenharias, as operações de guerra e não guerra (Ministério da Defesa, 2007; Beirão, 2013). Este campo é dominado pelas escolas militares de formação em todo o mundo e muito pouco foram estudados no âmbito civil, além de serem comumente parte da formação textual doutrinária.

Em contrapartida, tanto estudos de estratégia no que diz respeito às corporações e à própria guerra quanto estudos de logística, provocaram o avanço das ciências da administração no início do século (Rüdiger, 1990, p. 211). Do mesmo modo ocorreu quanto às ciências econômicas desde Adam Smith (Smith, 2001, cap. 5) até o pós-guerra, onde até a sua versão liberal tem expressa a relevância dos investimentos em defesa pelo Estado. Como parte dos estudos de recuperação econômica face à redução em investimentos de ordem bélica, também as ciências econômicas da defesa avançaram por meio do planejamento econômico, do orçamento de longo prazo, da otimização de gastos e das mais presentes teorias, como o dilema da manteiga versus canhões.

Por outro lado, esta componente civil da teoria econômica de caráter militar nos conduz à dualidade tecnológica, em que pese o uso cada vez mais intenso de investimentos de ordem militar com benefícios e propósitos de ordem civil. Neste ponto, faz-se necessária a ligação com os objetos das ciências aplicadas e exatas que compõem a parte ou o todo de um processo dual. Aqui estão incluídos estudos que distam sobre o papel da indústria, incluindo as suas relações com o Estado e em outros Estados.

A partir destes elementos, podemos também destacar outro ponto de intersecção possível a partir da guerra, ligados aos processos. Vigem processos do ponto de vista administrativo e logístico, mas também aqueles relacionados à formação de recursos humanos, em treinamento, capacitação, formação. Reverberam neste campo setores do conhecimento ligados à educação, à pedagogia ou à andragogia. Mas também são a este campo limítrofes os da psicologia e medicina, dotados pelas necessidades da guerra, dos limites físicos e psicológicos humanos. A estes campos associam-se os da sociologia, no que tange os conflitos causados por decisões sempre humanas, assim como as novas faces do exercício da defesa, em setores sociais e de apoio ao Estado.

Por último, não menos importante, as relações humanas não só tangenciam o campo das decisões e ações pertinentes ao campo da defesa, mas as experiências que reforçam ou afastam a incidência de determinadas teorias sobre a guerra e a paz. Por esta razão, não se pode observar o campo da defesa sem as interpretações históricas e as variações pertencentes à história da humanidade, onde se encontram os estudos sobre os regimes políticos, a incidência da democracia, suas instituições, os partidos políticos.

Diante disso, ao buscar nas bases os temas que foram enquadrados em revistas científicas ou grupos de pesquisa, encontramos o grupo de temas 
a seguir que, em nossa classificação compõem áreas tradicionais e campos híbridos da defesa, mesmo que eventualmente contidos em subáreas de diversas áreas. $\mathrm{O}$ resultado desta pesquisa está aqui apresentado na forma de um mapa de clusters, de modo a concentrar, de maneira não hierárquica, temas de caracterização dos estudos de defesa.

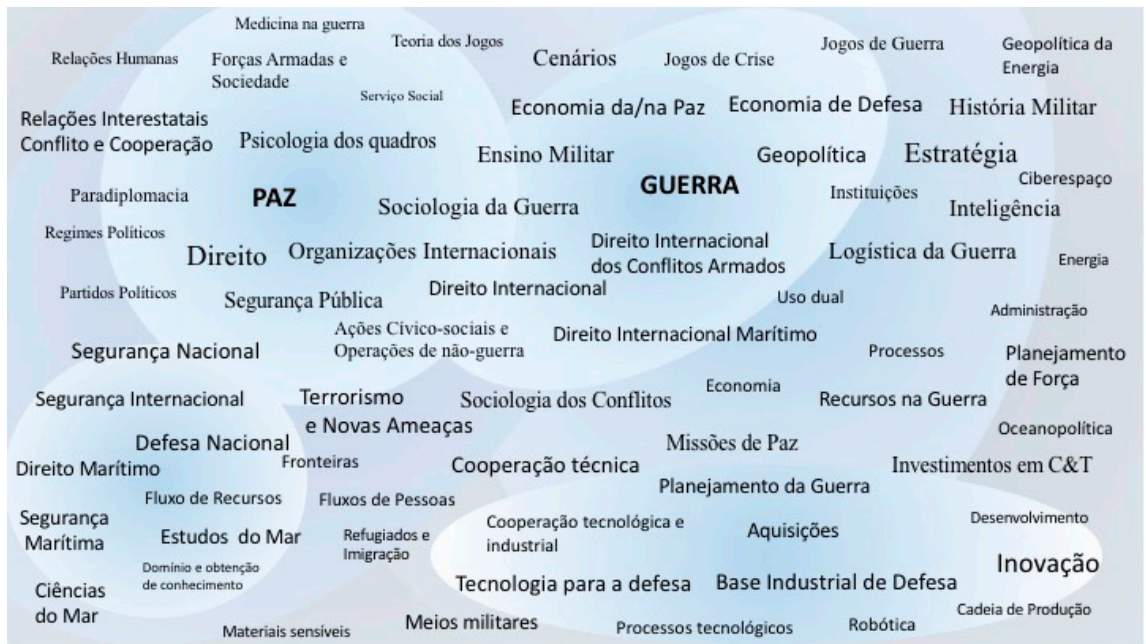

Figura 1 - Temas de caracterização dos estudos da defesa

Fonte: Elaborada pelos autores.

\section{POR UMA PROPOSTA DE REGIÕES HÍBRIDAS NO BRASIL}

A partir do tema da guerra é possível encontrar muitas variações, como se pode ver. Este trabalho foi feito com base em pesquisa nas principais bases de artigos científicos e nos grupos de pesquisa registrados no Diretório de Grupos de Pesquisa (DGP) do CNPq. Cabe notar que na estatística por área do DGP não há distinção da área de defesa entre as 77 áreas com indicadores, que também não distingue a área de relações internacionais, por exemplo. ${ }^{1}$

O efeito disto sobre Programas interdisciplinares é bastante notável, porque não aparecem como representativos de áreas de conhecimento consideradas estáveis, com grau razoável de distinção das demais. Em recente apresentação na forma de aula inaugural para o Programa de Pós-Graduação em Estudos Marítimos (PPGEM) da Escola de Guerra Naval, o presidente da Coordenação de Aperfeiçoamento do Ensino 
Superior (Capes), Carlos Nobre, acentuou a necessidade de que programas provenientes das ciências humanas e aplicadas sejam parte integrante de áreas estratégicas, como as ciências do mar, recentemente contempladas por um edital particular. Como demonstra o diagrama sobre o estado da pós-graduação na área de Ciências do Mar, o PPGEM - cujo mestrado profissional volta-se ao público de profissionais de setores como portos, logística, comércio, petróleo e jurídico - fora enquadrado no grupo de programas estratégicos, cujo escopo é o das ciências duras que não só as engenharias, mas as geociências, a biodiversidade e biotecnologia ou as ciências ambientais. Com isso, com base nos programas especializados da área de defesa, outra malha de áreas de conhecimento se abre, ampliando o escopo e o interesse pelos temas originalmente limitados à guerra.

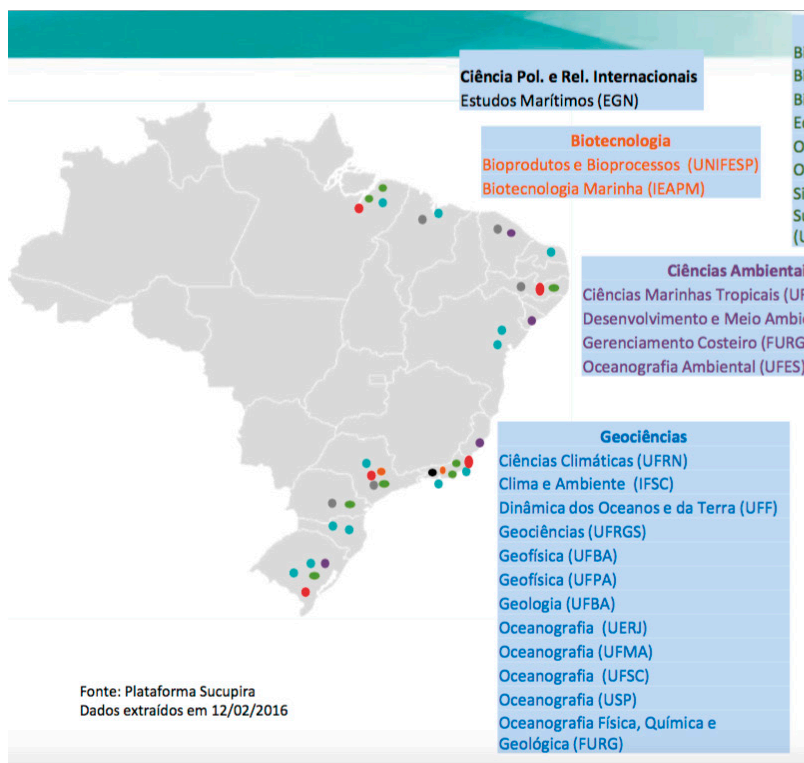

Biodiversidade

Biodiversidade e Biologia Evolutiva (UFRJ) Biologia Ambiental (UFPA) Biologia Marinha e Ambientes Costeiros (UFF) Ecologia Aquática e Pesca (UFPA) Oceanografia (UFPE)

Oceanografia Biológica (FURG)

Sistemas Costeiros e Oceânicos (UFPR)

Sustentabilidade de Eco. Costeiros e Marinhos (UNISANTA)

\section{to}

ais

Desenvolvimento e Meio Ambiente (FUFSE) Gerenciamento Costeiro (FURG) Oceanografia Ambiental (UFES)

Engenharias III

Engenharia Mecânica (UFPE)

Engenharia Naval (UFPA)

Engenharia Naval e Oceânica (USP)

Engenharia Oceânica (FURG) Engenharia Oceânica (UFRJ)

Zootecnia / Recursos Pesqueiros Aquicultura e Pesca (IP)

Engenharia de Pesca (UFC) Recursos Aquáticos e Pesca (UEMA) Recursos Pesqueiros e Aquicultura (UFRPE) Recursos Pesqueiros e Engenharia De Pesca (UNIOESTE)

37 programas em 25

IES:

25 ME $25 \mathrm{nn} 2 \mathrm{M}$.

Figura 2 - Mapa dos cursos de pós-graduação em Ciências do Mar

Fonte: Plataforma Sucupira.

Portanto, na qualificação do Programa de Pós-Graduação em Estudos Marítimos como sendo pertencente à área de Ciência Política e, apesar de sua submissão original para área multidisciplinar junto à Capes, há um reforço das humanidades nesta faixa de campos híbridos. De fato, um número 
maior de temas tende a concentrar-se em torno de clusters voltados para as humanidades, que progressivamente também são ampliadas em subáreas.

Por outro lado, um outro dado que pode demonstrar a imperfeição do sistema nos limites clássicos do entendimento de defesa é exemplificado na figura a seguir. Em uma busca feita na plataforma da SciELO, uma das maiores do mundo em acesso livre, foram encontrados somente 5 artigos com base nas palavras-chave defesa, militar e estratégia, demonstrando que mesmo dentro da defesa o campo mais tradicional não parece ser dominante do ponto de vista da indexação.

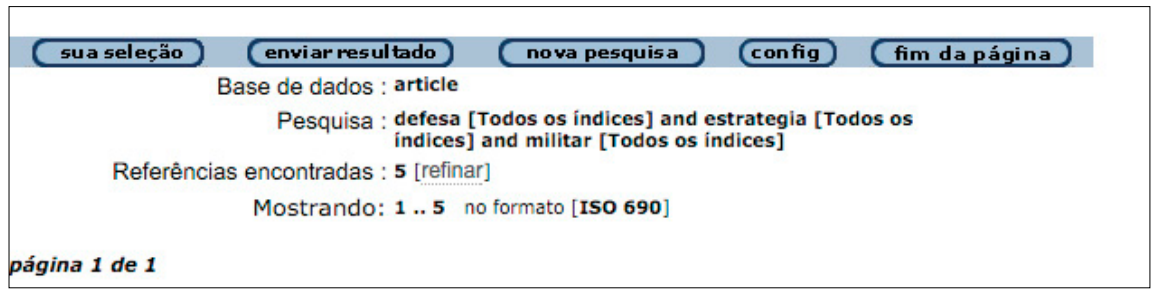

Figura 3 - Resultado de busca na base de dados SciELO

Fonte: Adaptado de SciELO.

Certamente, a criação da Associação Brasileira dos Estudos de Defesa, estimulada pela presença do Edital Pró-Defesa e Pró-Estratégia dentro dos últimos 10 anos não pode ser representada por este dado. Mas, se esta comunidade epistêmica é maior do que estes assuntos, como constituir empiricamente números confiáveis de sua representação? Na presença de indexadores incompletos e na observação por grandes e subáreas, a ciência brasileira está menos voltada ao impacto - que agrega valor aos campos híbridos - do que às áreas tais como definidas pelos órgãos de controle e fomento, onde também não há um modelo único vigente de parâmetros de indexadores de área.

Minha proposta é voltada para uma visão multidimensional e não hierárquica entre as partes, salvo pela detecção, em volume, do número de estudos que cada um representa, possibilitando por meio de análise de clusters quais os campos mais robustos. Em se pensando em fomento público, a lógica se reproduziria na maior parte das ocasiões, de forma a atender as demandas de maneira proporcional. O contrário disso poderia ser observado por meio de demandas induzidas, exclusivamente dotadas de recursos por objetivos estratégicos particulares e temporários, normalmente.

A importância da internacionalidade dos estudos desta área é, portanto, de ambos: a natureza dos estudos de defesa e seu alcance. O que procura- 
mos demonstrar é que os estudos de defesa não perderam a sua constituição inicial, de estudos da guerra, algo que nasce muito antes da criação dos Estados-Nação. Contudo, os estudos de defesa, que também tem algo de internacional, tem algo de particular, estritamente nacional, porque modelos cada vez mais se estendem nacionalmente e não são objeto apenas de produtores de conhecimento detentores de poder militar robusto.

Isso quer dizer que há um espaço particular de criação entre emerging countries como o Brasil que configura não só um mercado específico, mas uma conjuntura de desenvolvimento do conhecimento em defesa específica, conjuntural e baseada na comunidade epistêmica recém-criada. Há indícios de que estas comunidades de especialistas provocam o uso progressivo deste tipo de expertise em propostas de ordem política substantivas (Jacobs e Benjamin, 2005, p. 117).

Neste campo, a defesa, ganha contornos novos, provocados pela ascensão da incidência da ciência e tecnologia, da economia, da sociologia militar e das organizações. Também não é menor a contribuição da filosofia, da ética, da moral, do âmbito do direito, internacional, constitucional, ou direitos humanos. Cabe nesta interpretação a influência que comunidades epistêmicas podem ter sobre os processos, como partícipes de sua construção. Os estudos de defesa garantem isso, sobremaneira, fundamentalmente em uma década de crescimento expressivo dos investimentos em defesa.

Os contornos de seus temas principais, que evocam o estado de guerra, pré-guerra e pós-guerra, são interesses de Estado, embora reconfigurados no foco na estabilidade, reputação e defesa. Neste espaço, vigoram, pois, dois modelos de pensamento, muito recentemente aproximados, de origem civil e de origem militar. Alguns destes assuntos foram exercício de um ou outro grupo, dentre os que mais produziram estudos na área (Castro e Carreras, 2013; Gialdino, 2009).

\section{À GUISA DE CONCLUSÃO}

No Brasil, a presença de interesse na área acontece com a ascensão da área de relações internacionais, mas também, com a criação do Ministério da Defesa, com a reforma e constituição do próprio Estado, com o fomento dirigido pelos últimos governos. Assim se fizeram militares no ethos da Academia e civis no ethos dos militares. Ambos campos se abriram por uma conjuntura econômica favorável de progressiva internacionalização, uma comunidade epistêmica em formação - embora de relativa espontaneidade -, e algumas políticas públicas voltadas para isso, que encorajaram a reprodução dos trabalhos em defesa. 
Minha proposta para tempos de crise, em que pese a retenção dos investimentos em capital humano, formação, fomentos dirigidos, dentre outros, é a de que chega o melhor momento para a primeira parada, para que se observe qual o impacto de 10 a 15 anos de configuração do campo da defesa. Minhas observações sugerem a necessidade de estudos bibliométricos e de ordem estatística desta comunidade epistêmica e dos estudos de defesa que tenham como propósito observar como funcionalmente se constituíram estes clusters e sua produção.

Assim, hoje me constituo como parte disso. Ao olhar ao lado, me vejo neste campo, constituindo um novo pedaço dele com colegas de olhares com os quais compartilho os meus, em alguns - não somente um - destes clusters. Por meio destas zonas híbridas de conhecimento, a garantia de que suas faces estão inscritas em órbitas que escapam às grandes áreas em suas tradicionais lentes, mas que podem estar por elas absorvidas porque de valor estratégico, prioritariamente. Neste âmbito, como ordenar a este campo sem prejuízo aos demais e vice-versa é o desafio posto. Decerto, a divisão é sempre o pior caminho. Em um mundo regido pelo mercado, a demanda também assim se faz. Em um mundo regido pelas necessidades de um acerto entre a sociedade e como se deseja ver adiante, é inevitável pensar estes temas com afinco, com ardor, com desenvoltura. Construimo-nos.

\section{REFERÊNCIAS}

BEIRÃO, A. P. In Bello, Pax: a responsabilidade penal dos brasileiros em Operações de Paz. Tese (Doutorado em Direito). Programa de Pós-Graduação em Direito, Faculdade de Direito, Universidade do Estado do Rio de Janeiro, Rio de Janeiro,

BUZAN, B.; HANSEN, L. The evolution of international security studies. Cambridge: Cambridge University Press,

BUZAN, B.; HANSEN, L. Beyond the evolution of international security studies? Security Dialogue, 41 (6), p. 659-667,

CASTRO, C.; CARREIRAS, H. Qualitative methods in military studies. Research experiences and challenges. Oxon: Routledge,

DINIZ, E. Epistemologia, história e estudos estratégicos: Clausewitz versus Keegan. Contexto Internacional, Rio de Janeiro, v. 32, n. 1, p. 39-90, jun. Disponível em: $\quad<$ http://www.scielo.br/scielo.php?script=sci_arttext\&pi$\mathrm{d}=$ So 102-85292010000100002\&lng $=$ pt\&nrm=iso $>$.

FRENCH, P.; UEhling, T. H. K.; WeTtSTEIN. Studies in Epistemology. Minneapolis: University of Minnesota Press, GIALDINO, I. V. de. Ontological 
and Epistemological Foundations of Qualitative Research. Forum: Qualitative Social Research, v. 10, n. 2, art. 30, may 2009. Disponível em: <http://www.qualitative-research.net/index.php/fqs/article/view/1299/3163>.

HAAS, P. M. Introduction: epistemic communities and international policy coordination. International Organization, Cambridge, v. 46, n. 1, p. 1-35, 1992.

HANNAN, B. The riddle of the world: a reconsideration of Schopenhauer's philosophy. Oxford: Oxford University Press,

JACOBS, L. R.; BENJAMIN, I. Who influences U.S. foreign policy? Page Source: The American Political Science Review, v. 99, n. 1, p. 107-123, feb.

MINISTÉRIO DA DEFESA. Glossário das Forças Armadas. Disponível em: $<\leq<$ http://www.defesa.gov.br/arquivos/File/legislacao/emcfa/publicacoes/ md35_g_01_glossario_fa_4aed2007.pdf $>$.

MOREIRA, W. de S. Ciência e tecnologia militar: "política por outros meios"? Concurso de artigos sobre o Livro Branco de Defesa Nacional, Assessoria de Planejamento Institucional, Ministério da Defesa. Brasília: Ministério da Defesa, Disponível em: <http://www.defesa.gov.br/projetosweb/livrobranco/arquivos/ apresentacao-trabalhos/artigo-william-souza-moreira.pdf $>$.

PARSONS, T. Social systems and the evolution of action theory. New York: The Free Press,

POPPER, K. R. Objective knowledge, an evolutionary approach. Oxford: Oxford University Press,

RAWLS, J. A theory of justice. Cambridge: Harvard University Press,

RÜDIGER, P. The history of business administration and management education in the two Germanies - a comparative approach. The International Journal of Human Resource Management, v. 1, i. 2, p. 211-230,

SCHOPENHAUER, A. O mundo como representação. Livro III, Edição Acrópolis. Versão para E-book. 2001 [1819]. Disponível em: <http://www.ebooksbrasil. org/adobeebook/representacao3.pdf > .

SMITH, A. Wealth of Nations. Edited by C. J. Bullock. Vol. X. The Harvard Classics. New York: P. F. Collier \& Son, TURNER, J. H. The structure of sociological theory. 5. ed. Belmont: Wadsworth,

WALLACE, R. A.; WOLF, A. Contemporary sociological theory: continuing the classical tradition. 4. ed. Englewood Cliffs: Prentice Hall. 
1. Para acesso às estatísticas de Grupo de Pesquisa: <http://dgp.cnpq. $\mathrm{br} / \mathrm{dgp} /$ faces/relatorios/relIndicadoresGerais.jsf $>$.

\section{DA EPISTEMOLOGIA DOS ESTUDOS DE DEFESA E OS SEUS CAMPOS HÍBRIDOS"}

\section{RESUMO}

Este artigo trata da epistemologia dos estudos de defesa a partir de uma premissa de ordem evolucionária, onde o campo vem sendo configurado a partir de uma relação constante entre o que a sua comunidade vem propondo como sua parte constituinte e o que as instituições e políticas têm provocado nele. A primeira parte do artigo, pois, volta-se às bases filosóficas que constituem as bases da hipótese da existência de campos híbridos quantos aos estudos de defesa. A segunda parte do artigo é voltada para os diversos temas constituintes do campo da defesa, o estudo de sua natureza na qualidade de objeto científico e sua relação com os demais temas e disciplinas. Por fim, sugere-se a existência de estudos bibliométricos que sejam capazes de permitir uma observação mais precisa deste campo de estudos no Brasil, por meio de sua produção, interesses e arranjos institucionais.

Palavras-chave: Estudos de Defesa; Bibliometria; Ordem Evolucionária; Brasil.

\section{ABSTRACT}

This paper is based on the epistemology of defense studies from an evolutionary point of view, where the area has been configured from a constant relationship between what the community is proposing as its constituent part and what the institutions and policies have provoked. The first part of the article, therefore, proposes the philosophical ties that support the hypothesis of the existence of hybrid fields all around defense studies. The second part of the article is devoted to the various constituent themes of the defense studies area, the study of its nature as a scientific object and its relationship with other subjects and disciplines. Finally, it suggests the need of bibliometric studies that are able to allow a more accurate observation of this field in Brazil, through its production, interests and institutional arrangements.

Key-words: Defense Studies; Bibliometry; Evolutionary Order; Brazil.

Recebido em 14/03/2016. Aprovado para publicação em: 11/05/2016. 